\title{
Role of endoscopic management in synthetic sling/mesh erosion following previous incontinence surgery: a systematic review from European Association of Urologists Young Academic Urologists (YAU) and Uro-technology (ESUT) groups
}

\author{
Sulaiman Sadaf Karim ${ }^{1}$ - Amelia Pietropaolo ${ }^{1} \cdot$ Andreas Skolarikos $^{2} \cdot$ Omar Aboumarzouk $^{3} \cdot$ Panagiotis Kallidonis $^{4}$. \\ Thomas Tailly $^{5}$ • Vincent de Coninck ${ }^{6}$ • Etienne Xavier Keller ${ }^{7}$ - Bhaskar Kumar Somani ${ }^{1,8}$ (D)
}

Received: 17 May 2019 / Accepted: 8 August 2019 / Published online: 29 August 2019

(C) The Author(s) 2019

\begin{abstract}
Introduction and hypothesis Foreign body (FB) erosion is now recognized as a major long-term complication following previous incontinence surgery. The aim of our systematic review was to ascertain the outcomes of endoscopic management in synthetic sling/mesh erosion following previous gynaecological surgery.

Methods A systematic review in line with PRISMA and Cochrane guidelines was conducted for all English language articles between 1996 and December 2018 for all articles reporting on endoscopic surgical management for eroded FB following previous sling/mesh procedure for incontinence.

Results Our search produced 931 articles of which 20 articles (198 patients) were included in our review; 149 (75\%) had tensionfree vaginal tapes (TVT) or tension-free obturator tapes (TOT) as their initial procedure. The site of mesh erosion was the bladder in 134 patients $(68 \%)$ of which $12(6 \%)$ were in the bladder neck. Urethral mesh erosion was seen in 63 patients (32\%) across all studies.

The treatment of eroded mesh was by laser and endoscopic excision using an electrode loop or laparoscopic scissors in 108 (55\%) and 90 (45\%) patients respectively. The initial/final success rate with laser and endoscopic excision was $67 \% / 92 \%$ and $80 \% / 98 \%$ respectively. The overall complication rates were $24 \%$ and $28 \%$ in laser and endoscopic groups respectively of which $21 \%$ in each group were stress urinary incontinence.

Conclusions Endoscopic management of FB erosion is an effective minimally invasive technique with good outcomes and minimal morbidity. Management with the use of holmium laser is gaining momentum and could be attempted before open
\end{abstract}

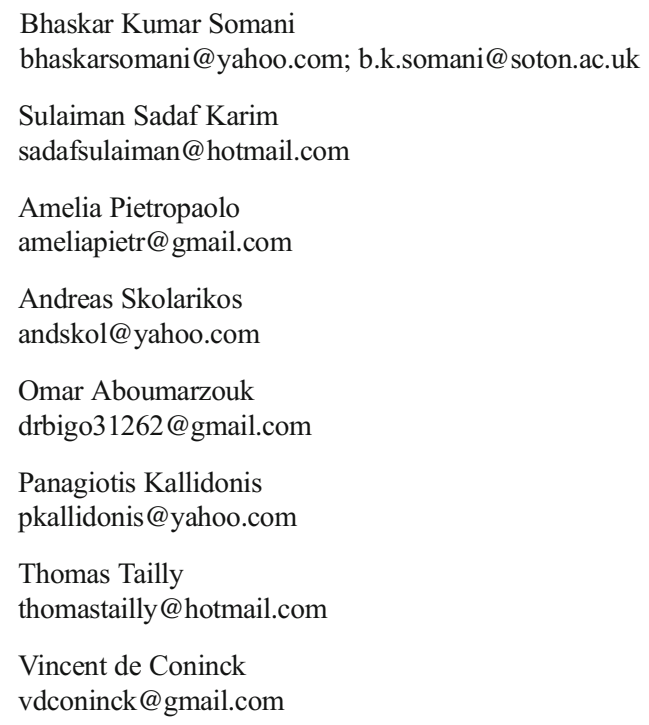

Etienne Xavier Keller etienne.xavier.keller@gmail.com

1 University Hospital Southampton NHS Trust, Southampton, UK

2 2nd Department of Urology, Sismanoglio Hospital, National and Kapodistrian University of Athens, Athens, Greece

3 Department of Urology, Queen Elizabeth University Hospital, Glasgow, UK

4 Department of Urology, University of Patras, Patras, Greece

5 University Hospitals Ghent, Ghent, Belgium

6 Department of Urology, AZ Klina, Brasschaat, Belgium

7 Department of Urolog, University Hospital Zurich, University of Zurich, Zurich, Switzerland

8 Department of Urology, University Hospital Southampton NHS Trust, Southampton SO16 6YD, UK 
surgical removal. There is a need for comparative data between open surgical excision and endoscopic excision to help better describe the patient's most likely to benefit from the endoscopic technique.

Keywords Endoscopic $\cdot$ Endoscopic removal $\cdot$ Endoscopy $\cdot$ Laser $\cdot$ Mesh $\cdot$ Sling $\cdot$ Tape $\cdot$ Incontinence $\cdot$ TVT $\cdot$ TOT $\cdot$ Mesh erosion

\section{Introduction}

Over the last 2 decades the treatment of stress urinary incontinence (SUI) has shifted to a midurethral sling (MUS) or a mesh-based bladder neck procedure [1]. Although the surgery was believed to be relatively safe, there has been a steep rise in the number of reported cases of their erosion into the lower urinary tract [2-10]. Mesh erosion was first reported in 2001 but is now recognized as a major long-term complication of MUS surgery with an incidence rate of between 0.6 and $5.4 \%$ $[2,11]$.

Although the true extent of mesh erosion seems to be under-reported, due to an alarming rise of reported cases in 2018, there was a temporary ban on all MUS procedures in the UK [12]. These erosions and related complications seem to have happened a few years after the initial surgery, with patients presenting with recurrent urinary tract infections (rUTIs), lower urinary tract symptoms (LUTS), recurrent SUI, haematuria and bladder or pelvic pain [13-15]. While open surgical excision via vaginal or abdominal route could be performed with urethrotomy and reconstruction in some cases [16], increasingly there are papers that suggest that they can be managed endoscopically.

Endoscopic management of eroded foreign body (FB) can be performed by intravesical resection of the mesh with electrode loop via a resectoscope or laparoscopic scissors [17-20], although it has been increasingly popular to use transurethral endoscopic resection using holmium laser [21]. These are minimally invasive approaches to a complex clinical problem.

The aim of our systematic review was to ascertain the outcomes of endoscopic management in synthetic sling/mesh erosion following previous incontinence surgery.

\section{Methods}

\section{Evidence acquisition}

Inclusion criteria:

1. Endoscopic surgical interventions for FB excision including laser procedures for adult patients who previously underwent synthetic sling/mesh procedure for incontinence.
2. Studies in English language reporting on a minimum of three patients.

\section{Exclusion criteria:}

1. Animal studies, case reports and laboratory studies

2. Open surgical interventions

3. Male sling procedures

\section{Search strategy and study selection}

A systematic literature search was conducted according to the Cochrane review and Preferred Reporting Items for Systematic Reviews and Meta-Analyses (PRISMA) protocol $[22,23]$. Medical subject headings (MeSH) terms used, but not limited to, were: 'endoscopic', 'laser', 'holmium', 'mesh', 'erosion', 'sling', 'TVT', 'tension-free vaginal tape', 'transobturator tape', 'TOT', 'urethral', 'transurethral', 'resection', 'removal', 'incontinence', 'surgical', 'incision' and 'intervention'. Boolean operators (AND, OR) were used to refine the search. The search strategy was conducted to find relevant articles from CINAHL (1996-December 2018), EMBASE (1996-December 2018), Ovid Medline (1996-December 2018), Cochrane Library (2018), Scopus (1996-December 2018), Clinicaltrials.gov, Google Scholar and individual urological journals. The search was confined to all English language articles between January 1996 and December 2018. All original studies were included and two reviewers (S.S and B.K.S.) identified all studies independently; discrepancies were resolved by mutual consensus. Where additional information or clarification was needed, the primary authors of the studies were contacted directly.

The PICO statement for this review is as follows: the population examined was adults who underwent synthetic sling procedures for incontinence. There was no comparative group, and the outcomes were to ascertain the results of endoscopic management following sling erosion.

\section{Data extraction and analysis}

The following variables were extracted from each study: journal and year of publication, study type, time period, presenting symptom, procedure type, time to presentation following initial surgery, location of mesh erosion, complications and their 
management, success rate and follow-up. We used the standard IUGA/ICS terminology for reporting mesh-associated complications in this manuscript [24]. Data were collated using Microsoft Excel (Microsoft Corp., Redmond, WA, USA), version 12.2.4.). There was a substantial degree of heterogeneity between studies in both design and reporting, and hence our analysis was limited to narrative synthesis and pooled analysis of mean results.

\section{Results}

Our search produced 931 articles of which 155 abstracts were reviewed and 20 articles (198 patients) met our inclusion criteria and were included in our final review (Fig. 1) [3-21, 25-27]. The removal of eroded sling/mesh was done by laser incision in nine studies, endoscopic approach using transurethral resection (TUR) or cutting it with scissors in seven studies and a mixture of the above two techniques in the remaining four studies (Table 1). In the reported studies, 108 (55\%) patients were treated exclusively with laser excision and 90 (45\%) were managed by intravesical resection of the mesh with electrode loop via a resectoscope, laparoscopic scissors or forceps.

\section{Demographics and clinical presentation}

All of the included articles were retrospective case series and reported on 3-23 patients each. The mean age across studies varied from 48 to 68 years, and 149 (75\%) had tension-free vaginal tapes (TVT) or transobturator tapes (TOT) as their initial procedure. The journal, year of publication, level of evidence and time to presentation after their original surgery are reported in Table 1.

Voiding lower urinary symptoms (LUTS) was the most commonly reported symptom (17 studies). Other symptoms included recurrent UTIs (11 studies), haematuria (9 studies), SUI (6 studies), urge incontinence (UI) (5 studies), dyspareunia (7 studies), dysuria (4 studies) and pelvic pain (3 studies). Mesh-associated stones were a common finding across the literature in patients with urinary mesh erosion. The median time to presentation after their initial surgery was 34.5 months (range: $1-247$ months).

\section{Site of mesh erosion}

The site of mesh erosion was the bladder in 134 patients (68\%), of which $12(6 \%)$ were in the bladder neck. Urethral mesh erosion was seen in 63 patients (32\%) across all studies.

\section{Outcomes with laser incision}

One hundred eight $(55 \%)$ patients were treated exclusively with laser incision (Table 2). The initial and final success rate with this technique was 67\% (72 patients) and 92\% (99 patients) respectively. Of these patients, 27 needed further laser procedures, with $18(17 \%)$ needing one additional procedure, $6(5.6 \%)$ needing two additional procedures and $3(2.8 \%)$ needing multiple $(>2)$ procedures. Of the remaining nine patients, eight $(7 \%)$ needed vaginal surgery to remove a mesh remnant and one needed open cystotomy to remove the eroded mesh.

Post-operative complications were seen in 26 (24\%) patients. These included SUI in $23(21 \%)$, urinary tract infection in $2(1.9 \%)$ and voiding difficulty in 1 patient. There were no
Fig. 1 PRISMA flowchart of the included studies
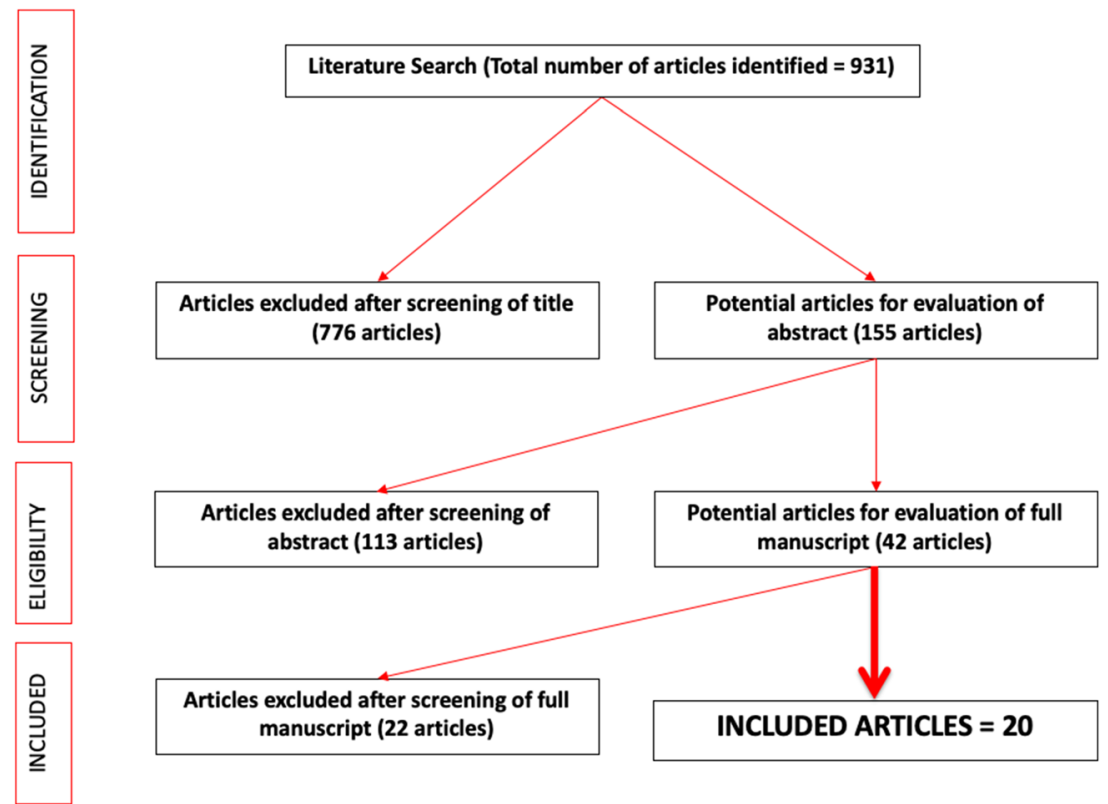


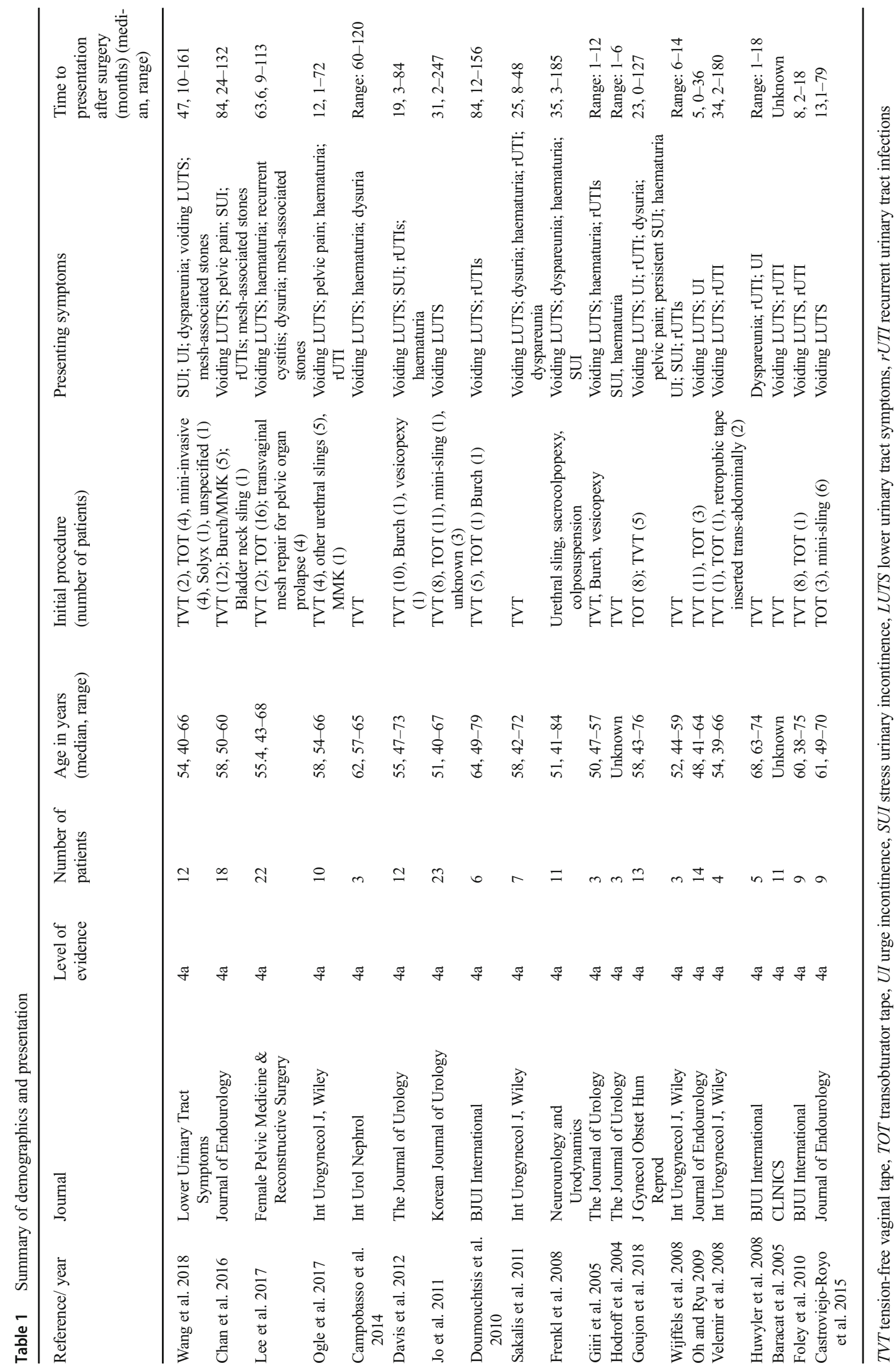




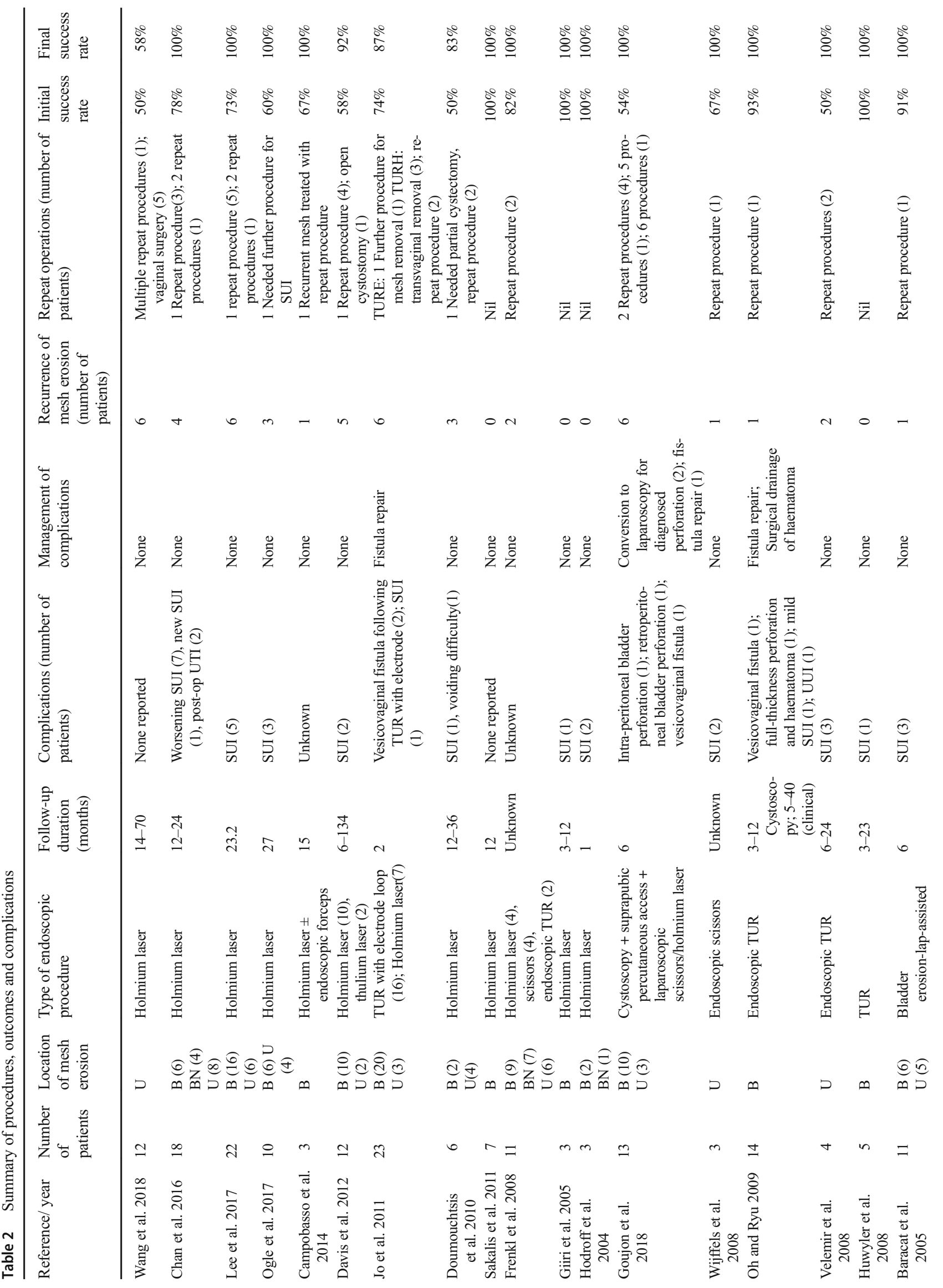


other reported major complications such as fistula formation or intra-operative perforation.

\section{Outcomes with endoscopic procedure using resectoscope/electrode loop or laparoscopic scissors/forceps}

Ninety (45\%) patients were managed using TUR with an electrode loop or laparoscopic scissors/forceps. The initial and final success rates with this technique were $80 \%$ ( 72 patients) and $98 \%$ ( 88 patients) respectively. Of these patients, 16 needed further endoscopic procedures, with $10(11 \%)$ needing one additional procedure, $4(4 \%)$ needing two additional procedures and $2(2 \%)$ needing multiple $(>2)$ procedures. The remaining two patients needed open surgery to remove the eroded mesh.

Post-operative complications were seen in $25(28 \%)$ patients. These included SUI in 19 (21\%), vesicovaginal fistula in $3(3 \%)$, intra-peritoneal bladder perforation needing a further procedure in $2(2 \%)$ and retroperitoneal bladder perforation in 1 patient.

\section{Discussion}

\section{Meaning of the study}

While there is increasing concern about the growing numbers of mesh or sling erosions, all types of surgical intervention carry a risk of morbidity. Bearing in mind the limitations of our review, which is based on retrospective case series, endourological techniques show excellent success rates of $>$ 90\% with endoscopic techniques using laser excision or endoscopic resection, although 20-25\% patients may need more than one endoscopic procedure to achieve this. Considering the lack of an open comparator group and reporting bias, the real success rate could be much lower. While the complication rates seem to be 24-28\%, one would argue that the majority of these were SUI, which is expected as an outcome of mesh removal irrespective of whether it is done endoscopically or as an open approach, and this may not really count as a complication but probably as an expected outcome of the mesh removal procedure.

\section{Outcomes with practical considerations related to mesh erosion}

Polypropylene meshes play a huge role in the minimally invasive repair of pelvic organ prolapse (POP) and SUI [28]. However, this also presents complications of mesh misplacement, migration or erosion [4]. FB erosion following urinary incontinence surgery can be a late complication and may be associated with the material used, operating surgeon's 
expertise and technical difficulties at the time of initial surgery $[4,13]$. Unrecognized bladder perforation during trocar insertion can also cause mesh erosion and may present as a late complication [3].

Often presenting with voiding LUTS, recurrent UTIs and haematuria, other symptoms identified in our review included SUI, urge incontinence (UI), dysuria and dyspareunia. The median time of presentation after surgery was 34 months, and a low index of suspicion is necessary in these patients with a previous history of incontinence-related procedures. This delay in presentation can be due to vague non-specific symptoms and a lack of standardized follow-up [29]. A high index of suspicion is often needed, and a detailed evaluation is necessary often with a cystoscopy to examine the LUT, which can help to confirm the diagnosis. Mesh-associated stones are not uncommon and the presence of stones should raise a strong suspicion of mesh erosion.

Our review of the literature, which is the largest to date, shows that endoscopic management is a feasible and effective option in managing FB erosion. Holmium laser was associated with an overall success rate of $92 \%$ and endoscopic management with resectoscope/laparoscopic scissors had a success rate of $98 \%$. The most common complication was SUI in both groups; however it was largely unclear whether this was de novo or a pre-existing complaint. SUI following removal of mesh surgery can be due to a failed incontinence procedure but equally mesh erosion can damage the urethra and sphincter resulting in scarring. The management of this can be complicated and may need reconstructive surgery with urethroplasty to help improve functional outcome [4]. Endoscopic management with resectoscope and electrode loop and the use of laparoscopic scissors was associated with vesicovaginal fistula in three patients and bladder perforation in two patients. It is worth noting that the laser approach allowed for decreased post-operative morbidity and complications, decreased inpatient hospital stay and good functional outcomes [7]. However, the outcomes of the two approaches may not be directly comparable because of different populations, variation in techniques and different surgical expertise.

\section{Implications for clinical practice}

The duration of follow-up varied across all studies and ranged from 1 to 134 months. We suspect it is important for all patients to have long-term outpatient-based follow-up and perhaps have a vaginal speculum examination along with a flexible cystoscopy $6-12$ months post-operatively to pick up any early signs of mesh erosion [30]. This could be repeated if there are any obvious symptoms or a clinical suspicion of erosion. Presence of bladder or urethral stones especially if adherent to the musoca suggests an erosion. The patients should also be counselled to return for a review if they develop persistent or worsening urinary symptoms. Patients at high risk of potential mesh-related complications seem to be those who have a combined mesh for POP repair and SUI mesh sling [30]. In a study of 41,604 women in the US who underwent transvaginal mesh repair for POP with or without concurrent sling for SUI, transvaginal POP repair without mesh but with concurrent sling or a sling for SUI only and a follow-up period of 1-year, the risk of erosion was $2.7 \%$ and the risk of repeated surgery with concomitant erosion was $2.1 \%$ in the POP repair with mesh plus sling group [30]. The SUI sling group on its own had an erosion rate of $1.5 \%$ and a risk of repeated surgery with concomitant erosion of $1.6 \%$. Similarly, concomitant hysterectomy and hypertension were also associated with mesh erosions [31]. In high-risk patients the index of FB erosion and early counselling and appropriate management should be made available.

\section{Strengths, limitations and areas of future research}

In this review we adhere to the methodological approach of the Cochrane guidelines and PRISMA checklist. It summarizes the role of endoscopic management of mesh erosions including the use of both laser and transurethral resection and gives an overview of the success rate and complications with this technique, although all papers included in our review were retrospective case series and hence prone to bias. Furthermore, there was a lot of heterogeneity in the reported studies making it challenging to do a formal meta-analysis. There are some limitations to our systematic review. Individual studies did not have a standardized management or follow-up, and this was left to the individual surgeon and centre based on their expertise and patient presentation. Given that data collection and reporting were not standardized either, it was difficult to compare or combine outcomes.

A recent study looked at the reporting outcome measures in trials on synthetic mesh procedures for POP and concluded that urgent action is needed to improve the quality of research in this field [32]. Of the 71 randomized trials, 24 different types of mesh were identified. These trials reported on 110 different outcomes and 60 outcome measures. Clinically important measures such as erosion, pain and dyspareunia were reported in 40,29 and 25 trials respectively. They recommend developing and implementing a minimum standardized data set, which forms the core outcomes of this procedure. Another systematic review on the type of synthetic material used found that polyester sling material caused the highest rates of vaginal erosion [33]. The US Food and Drug Administration (FDA) has ordered all manufacturers of surgical mesh intended for transvaginal repair of prolapse (cystocoele) to stop selling and distributing the product [34]. Given the scale of mesh erosions and the public outcry associated with it, perhaps a national registry of all sling or mesh procedures should be the way ahead, where every case is registered and the core outcome measures including the type of material used, surgical 
technique, complications and follow-up are mandatory. This should be protocol based and have outcome measures which are standardized and hence comparable.

\section{Conclusions}

Endoscopic management of FB erosion is an effective minimally invasive technique with good outcomes and minimal morbidity, which uro-gynaecologists or endourologists might be able to offer as a treatment option. Management with the use of holmium laser is gaining momentum and could be attempted before open surgical removal. There is a need for comparative data between open surgical excision and endoscopic excision to help better describe the patient's most likely to benefit from the endoscopic technique.

\section{Compliance with ethical standards}

\section{Conflicts of interest None.}

Open Access This article is distributed under the terms of the Creative Commons Attribution 4.0 International License (http:// creativecommons.org/licenses/by/4.0/), which permits unrestricted use, distribution, and reproduction in any medium, provided you give appropriate credit to the original author(s) and the source, provide a link to the Creative Commons license, and indicate if changes were made.

\section{References}

1. Oliphant SS, Wang L, Bunker CH, Lowder JL. Trends in stress urinary incontinence inpatient procedures in the United States, 1979-2004. Am J Obstet Gynecol. 2009;200:521.e1-6.

2. Koelbl H, Stoerer S, Seliger G, Wolters M. Transurethral penetration of a tension-free vaginal tape. BJOG. 2001;108:763-5.

3. Giri SK, Drumm J, Flood HD. Endoscopic holmium laser excision of intravesical tension-free vaginal tape and poly-propylene suture after anti-incontinence procedures. J Urol. 2005;174(4 Pt 1):13067.

4. Frenkl TL, Rackley RR, Vasavada SP, Goldman HB. Management of iatrogenic foreign bodies of the bladder and urethra following pelvic floor surgery. Neurourol Urodyn. 2008;27:491-5.

5. Jo D-J, Lee Y-S, Oh T-H, Ryu D-S, Kwak K-W. Outcomes of transurethral removal of intravesical or intraurethral mesh following midurethral sling surgery. Korean J Urol. 2011;52:829-34.

6. Hodroff M, Portis A, Siegel SW. Endoscopic removal of intravesical polypropylene sling with the holmium laser. J Urol. 2004;172(4 Pt 1):1361-2.

7. Sakalis VI, Gkotsi AC, Triantafyllidis A, Giouris A, Charalambous S. Transurethral holmium laser intravesical tape excision following TVT procedure: results from seven patients in a 12-month followup. Int Urogynecol J. 2012;23:769-77.

8. Davis NF, Smyth LG, Giri SK, Flood HD. Evaluation of endoscopic laser excision of polypropylene mesh/sutures following antiincontinence procedures. J Urol. 2012;188:1828-32.

9. Campobasso D, Cerasi D, Fornia S, Meli S, Ferretti S, Cortellini P. Endoscopic holmium laser management of tension-free vaginal tape eroded into the bladder. Int Urol Nephrol. 2014;46:1507-10.
10. Ogle CA, Linder BJ, Elliott DS. Holmium laser excision for urinary mesh erosion: a minimally invasive treatment with favorable longterm results. Int Urogynecol J. 2015;26:1645-8.

11. Novara G, Galfano A, Boscolo-Berto R, et al. Complication rates of tension-free midurethral slings in the treatment of female stress urinary incontinence: a systematic review and meta-analysis of randomized controlled trials comparing tension-free midurethral tapes to other surgical procedures and different devices. Eur Urol. 2008;53:288.

12. Immediate stop to NHS mesh operations [Internet]. BBC News. 2019 [cited 21 December 2018]. Available from: https://www.bbc. co.uk/news/health-44763673.

13. Baracat F, Mitre AI, Kanashiro H, Montellato NID. Endoscopic treatment of vesical and urethral perforations after tension-free vaginal tape (TVTÒ) procedure for female stress urinary incontinence. Clinics. 2005;60:397-400.

14. Velemir L, Amblard J, Jacquetin B, Fatton B. Urethral erosion after suburethral synthetic slings: risk factors, diagnosis, and functional outcome after surgical manage- ment. Int Urogynecol J Pelvic Floor Dysfunct. 2008;19:999-1006.

15. Huwyler M, Springer J, Kessler TM, Burkhard FC. A safe and simple solution for intravesical tension-free vaginal tape erosion: removal by standard transurethral resection. BJU Int. 2008;102: $582-5$.

16. Volkmer BG, Nesslauer T, Rinnab L, et al. Surgical intervention for complications of tension-free vaginal tape procedure. J Urol. 2003;169:570.

17. Wijffels SAM, Elzevier HW, Nijeholt AABLA. Transurethral mesh resection after urethral erosion of tension-free vaginal tape: report of three cases and review of literature. Int Urogynecol J. 2008;20:261-3.

18. Oh T-H, Ryu D-S. Transurethral resection of intravesical mesh after midurethral sling procedures. J Endourol. 2009;23:1333-7.

19. Foley C, Patki P, Boustead G. Unrecognized bladder perforation with mid-urethral slings. BJU Int. 2010;106:1514-8.

20. Castroviejo-Royo F, Rodríguez-Toves LA, Martínez-Sagarra-Oceja JM, Conde-Redondo C, Mainez-Rodríguez JA. Outcomes of transurethral resection (TUR) of intravesical mesh after suburethral slings in the treatment of urinary stress incontinence. Actas Urol Esp. 2015;39:183-7.

21. Wang C, Zimmern P, Lemack G. Long-term results of transurethral endoscopic excision using the holmium laser for urethral perforation of synthetic slings. Low Urine Tract Symptoms. 2018. https:// doi.org/10.1111/luts.12226.

22. Higgins JP, Green S, editors. Cochrane handbook for systematic reviews of interventions. Chichester: Wiley; 2008. Accessed 03 Mar 2019.

23. Moher D, Liberati A, Tetzlaff J, Altman DG, The PRISMA Group. Preferred reporting items for systematic reviews and meta-analyses: the PRISMA statement. PLoS Med. 2009;6(7):e1000097.

24. Haylen BT, Freeman RM, Swift SE, et al. An International Urogynecological Association (IUGA)/International Continence Society (ICS) joint terminology and classification of the complications related directly to the insertion of prostheses (meshes, implants, tapes) \& grafts in female pelvic floor surgery. Int Urogynecol J. 2011;22:3-15. https://doi.org/10.1007/s00192-0101324-9.

25. Chan G, Mamut A, Martin P, et al. Holmium:YAG laser ablation for the management of lower urinary tract foreign bodies following incontinence surgery: a case series and systematic review. J Endourol. 2016;30(11):1252-61.

26. Doumouchtsis S, Lee F, Bramwell D, Fynes M. Evaluation of holmium laser for managing mesh/suture complications of continence surgery. BJU Int. 2011;108(9):1472-8.

27. Lee $\mathrm{C}, \mathrm{Ku} \mathrm{J}$, Lee $\mathrm{K}$, et al. Clinical application of a transurethral holmium laser excision of exposed polypropylene mesh at lower 
urinary tract. Female Pelvic Medicine \& Reconstructive Surgery. 2018;24(1):26-31.

28. Nolfi AL, Brown BN, Liang R, et al. Host response to synthetic mesh in women with mesh complications. Am J Obstet Gynecol. 2016;215(2):206.e1-206.e2068. https://doi.org/10.1016/j.ajog. 2016.04.008.

29. Goujon E, Jarniat A, Bardet F, Bergogne L, Delorme E. Retrospective study on the management and follow-up of 18 patients with a mid-urethral sling penetrating the urethra or bladder. J Gynecol Obstet Hum Reprod. 2018;47(7):289-97. https://doi.org/ 10.1016/j.jogoh.2018.05.007.

30. Chughtai B, Barber MD, Mao J, et al. Association between the amount of vaginal mesh used with mesh erosions and repeated surgery after repairing pelvic organ prolapse and stress urinary incontinence. JAMA Surg. 2017;152(3):257-63.

31. Cheng YW, Su TH, Wang H, et al. Risk factors and management of vaginal mesh erosion after pelvic organ prolapse surgery. Taiwan $\mathrm{J}$ Obstet Gynaecol. 2017;56(2):184-7.
32. De Mattos Lourenco TR, Pergialiotis V, Duffy JMN, et al. A systematic review on reporting outcomes and outcome measures in trials on synthetic mesh procedures for pelvic organ prolapse: urgent action is needed to improve quality of research. Neurourol Urodyn. 2019;38(2):509-24.

33. Nalliah $\mathrm{S}$, Teng $\mathrm{H}$, Chong $\mathrm{XY}$, et al. Incidence of vaginal erosion with different synthetic materials for suburethral sling in the treatment of stress urinary incontinence: a systematic review. Med J Malaysia. 2018;73(3):147-53.

34. https://www.fda.gov/medical-devices/implants-and-prosthetics/ urogynecologic-surgical-mesh-implants. Accessed 28 Apr 2019.

Publisher's note Springer Nature remains neutral with regard to jurisdictional claims in published maps and institutional affiliations. 